\title{
Previously Asymptomatic Ruptured Tubal Ectopic Pregnancy at over 11 Weeks Gestation
}

\author{
Sukhamoy Barik ${ }^{1}$, Sudipta Bhattacharya ${ }^{2}$, Asish Pal ${ }^{3}$, Papia Dutta ${ }^{4}$ \\ ${ }^{1}$ Department of Obstetrics and Gynaecology, B N Bose Hospital, Barrackpore, West Bengal, India. \\ ${ }^{2}$ Department of Obstetrics and Gynaecology, B N Bose Hospital, Barrackpore, West Bengal, India. \\ ${ }^{3}$ Department of Anaesthesia, B N Bose Hospital, Barrackpore, West Bengal, India. ${ }^{4}$ Department of \\ Obstetrics and Gynaecology, ESI, Maniktala, West Bengal, India.
}

\section{PRESENTATION OF CASE}

A 23-year-old, newly married female, G1P0 at $113 / 7$ weeks' gestation, presented to the emergency department in B N Bose Hospital, Sub-Divisional Hospital, in West Bengal, complaining of sudden suprapubic and abdominal tenderness. It began after a single episode of nausea and vomiting earlier that morning. She described the pain as cramping and had noticed some spotting as well. She denied having any prior symptoms, and also denied significant medical, surgical or obstetric history. Her vital signs in the emergency department were- pulse-110 bpm, BP- 90/60, and Sp02 94\% with room air.

On physical examination, the patient was distressed. Her abdomen was nondistended but diffusely tender to palpation in the suprapubic region, worse on the right side. She had guarding with no rigidity. Laboratory results were positive for anaemia (haemoglobin- $5 \mathrm{gm} / \mathrm{dL}$ ) and positive urine pregnancy test.

USG revealed right ovarian haemorrhagic cyst with moderate ascites. No intrauterine sac demonstrated.

Decision of laparotomy was taken as patient's condition was deteriorating. Surgical evaluation demonstrated significant haemoperitoneum and ruptured right sided tubal pregnancy at ampulla with an exposed foetus. Right sided salpingectomy was done, and the pregnancy was removed. Estimated blood loss was $3500 \mathrm{~mL}$ necessitating 4 units blood product administration. The patient was monitored closely and she was discharged home on post-operative day five.

Ectopic pregnancy is a life-threatening condition affecting up to $2 \%$ of gestations. Implantation in the fallopian tube is most common, with symptoms typically presenting earlier for gestations in the ampulla and isthmus compared with the cornua and non-tubal sites. Isthmic rupture usually occurs at 6-8 weeks, the ampullary one at $8-12$ weeks and the interstitial one at about 4 months.
Corresponding Author: Dr. Sukhamoy Barik, Basundhara Apartment, \#9 M C Garden Road, Kolkata-700030, Dum Dum, West Bengal, India. E-mail: sukhamoy83@gmail.com

DOI: $10.14260 / j e m d s / 2020 / 355$

Financial or Other Competing Interests: None.

How to Cite This Article:

Barik S, Bhattacharya S, Pal A, et al. Previously asymptomatic ruptured tubal ectopic pregnancy at over 11 weeks gestation. J. Evolution Med. Dent. Sci. 2020;9(20):1622-1624, DOI: $10.14260 / \mathrm{jemds} / 2020 / 355$

Submission 17-03-2020,

Peer Review 01-05-2020

Acceptance 07-05-2020,

Published 18-05-2020.

\section{(i)}



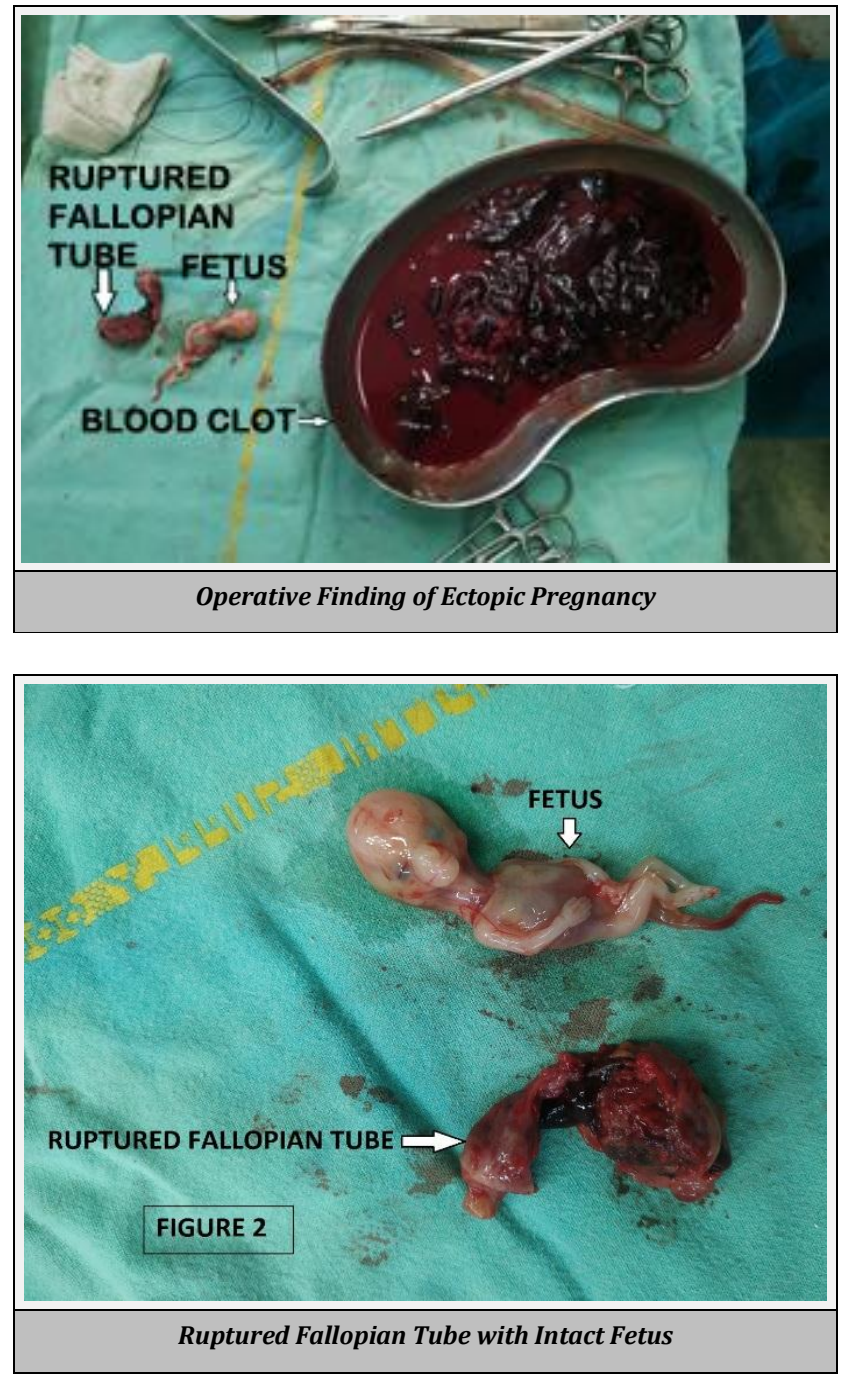

\section{DISCUSSION}

Ectopic pregnancy affects up to $2 \%$ of gestations. ${ }^{[1,2]}$ While fallopian tube involvement is the most common, abdominal, cervical, ovarian, and caesarean section scar implantation have also been reported.[1],[2],[3],[4] Risk factors predisposing women to ectopic pregnancies hinge on disrupted fallopian tube anatomy.[1] This can be congenital, but often results from prior tubal ectopic pregnancies, elective abortions, prior miscarriages, and sexually transmitted infections.[1,5,6] Smoking and advanced maternal age, Intrauterine device, post coital pill, ART have also been implicated.[6]While diagnostic technique has improved over time, it is not without error.

Furthermore, using reported risk factors and presenting symptoms to monitor likelihood of ectopic pregnancy has its limitations as well. This patient was previously asymptomatic and no risk factor indicating that close monitoring of individuals at low to moderate risk can be equally as important.

Management of ectopic pregnancy, like diagnosis, has been enhanced by medical advances and yet carries its own risks. Gestations that sonographically appear as extrauterine lesions measuring smaller four cm without any fetal cardiac activity, serum BhCG <3000 IU/L, No intraabdominal haemorrhage in haemodynamically stable women may be amenable to the non-invasive expectant or medical management; however, in women who are hemodynamically unstable, have a beta-HCG greater than $3000 \mathrm{IU} / \mathrm{L}$, or have a pregnancy that sonographically appears as an extrauterine lesion larger four $\mathrm{cm}$, surgical management is recommended.[5] Laparoscopic salpingectomy or salpingotomy are the preferred surgical options in hemodynamically stable women as these options are less invasive than open surgery; however, conversion to open surgery is warranted if the ectopic pregnancy cannot be adequately excised and bleeding is uncontrolled.[1] Salpingotomy carries an increased risk of persistent trophoblast compared to salpingectomy; thus, it is important to monitor beta-HCG following surgery.[2,7]

The extensive hemoperitoneum experienced in this cases demonstrates well the life-threatening nature of ectopic pregnancy and the increased risk associated with higher gestational age. Ectopic pregnancy, in general, causes the largest morbidity and mortality in early pregnancy. ${ }^{[8]}$ Not only can it cause future pain and impaired fertility, but it can also more acutely cause intraperitoneal bleeding and anemia, potentially necessitating blood transfusion.[2,9] A report published in 2016 stated that approximately 5\% of all maternal deaths are directly connected to ectopic pregnancy, with over half of those cases not being evaluated for the diagnosis. [2]

The American College of Obstetricians and Gynecologists recommends that pregnant patients receive a minimum of one standard ultrasound exam, typically between 18 and 22 weeks of gestation.[10] It notes that first-trimester exams are not considered standard, given the inability to distinguish detailed fetal anatomy during this period. This case series highlights the importance of considering routine, early first-trimester ultrasound to determine implantation site despite lack of risk factors or lack of symptoms. It also elucidates an opportunity for enhanced patient education regarding the potential for ectopic pregnancy, the associated signs and symptoms, and the overall importance of early and consistent prenatal care for prompt detection and reduction of maternal morbidity and mortality.

\section{CONCLUSIONS}

Ectopic pregnancy is a potentially fatal emergency condition if early diagnosis is missed. The presentations vary widely from being asymptomatic to haemodynamically compromised. The multitude of presentations to the primary care physician on first contact can be misleading in the absence of a high index of suspicion. So proper clinical examination, high index of suspicion, investigation including serial serum BhCG and TVS in early weeks of pregnancy are of utmost important factors to diagnose ectopic pregnancy. Early intervention can save the life of a mother.

\section{REFERENCES}

[1] Cunningham F, Leveno KJ, Bloom SL, et al. Williams Obstetrics. Chap - 19. Ectopic pregnancy. 24th edn. New York: McGraw-Hill Publisher 2013.

[2] Madhra M, Otify M, Horne AW. Ectopic pregnancy. Obstet Gynaecol Reprod Med 2017;27:8. 
[3] Robertson JJ, Long B, Koyfman A. Emergency medicine myths: ectopic pregnancy evaluation, risk factors and presentation. J Emerg Med 2017;53(6):819-28.

[4] Mahapatro AK, Shankar K, Varma T. Caesarean scar ectopic pregnancy: report of two cases. J Clin Diagn Res 2016;10(5):QD05-6.

[5] Odejinmi F, Huff KO, Oliver R. Individualisation of intervention for tubal ectopic pregnancy: historical perspectives and the modern evidence based management of ectopic pregnancy. Eur J Obstet Gynecol Reprod Biol 2017;210:69-75.

[6] Bouyer J, Coste J, Shojaei T, et al. Risk factors for ectopic pregnancy: a comprehensive analysis based on a large case-control, population-based study in France. Am J Epidemiol 2003;157(3):185-94.
[7] D'Hooghe T, Tomassetti C. Surgery for ectopic pregnancy: making the right choice. Lancet 2014;383(9927):1444-5.

[8] Adams JG, Barton ED, Collings J, et al. Emergency medicine: clinical essentials. Disorders of early pregnancy. $2^{\text {nd }}$ edn. Philadelphia, PA: Elsevier/ Saunders 2013.

[9] Akaba GO, Ekele BA, Onafowokan 0, et al. Comparative analysis of morbidity and mortality due to ectopic pregnancy at a tertiary care hospital in Nigeria over two study periods. Int J Gynecol Obstet 2015;128(1):76-7.

[10] American College of Obstetricians and Gynecologists Ultrasound Exams. (Accessed April 17, 2018). https://www.acog.org/Patients/FAQs/UltrasoundExams 